\title{
Multislice Computed Tomography Imaging with Clinical Outcome in Inflammatory Appendiceal Masses
}

VARSHA RANGANKAR, ABHIJIT PAWAR, ADITI DONGRE, BRIG HARIQBAL SINGH

\section{ABSTRACT}

Introduction: Appendicular lump is a serious complication of appendicitis presenting clinically with right iliac fossa mass which has a range of differential diagnosis. Diagnosis of complicated appendicitis and appendicular lump is often difficult and challenging. Computed tomography imaging has an important role in diagnosis and differentiation of appendicular mass.

Aim: The purpose of this study was to assess the computed tomography imaging features of inflammatory appendiceal masses.

Materials and Methods: Multislice computed tomography (MSCT) images of total 56 patients of inflammatory appendiceal masses were retrospectively analyzed during the period of October 2011 to November 2015. Contiguous axial CT sections obtained before and after injection of iodinated intravenous contrast medium were analyzed. Oral and rectal contrast was also administered in all patients to opacify the bowel. Images were evaluated for inflammed appendix, appendicolith, extraluminal fluid collections, inflammatory phlegmon formation, extraluminal air, fat inflammation, fascial thickening, bowel aggregation, bowel wall thickening and enlarged lymph nodes.

Results: Fifty six patients of inflammatory appendiceal masses with mean age 41-50 years were identified which included $31(55 \%)$ men and $25(45 \%)$ women. The inflamed appendix could be identified in the inflammatory mass in 22 cases (39\%) with appendicolith seen in 12 cases (21\%). Extraluminal fluid collections were seen in 35 patients $(62.5 \%)$ with walled-off collections with enhancing walls labelled as abscesses were seen in 26 cases $(46 \%)$. Inflammatory phlegmonous mass was seen in 38 cases ( $68 \%$ ) while adjacent ileocecal wall thickening was seen 22 cases (39\%). Omental thickening and fat inflammation was present in 46 out of 56 cases (82\%) with adjoining aggregated small bowel loops were seen in 36 cases $(64 \%)$. Extraluminal air pockets were seen in 31 patients $(55 \%)$. Out of 35 patients with extraluminal fluid collections, 24 were drained percutaneously under image guidance which included 15 patients labelled as abscess. In five patients, the collections were small in size and resolved with conservative treatment. Nineteen patients including four patients with large abscesses and pelvic extensions underwent laparotomy.

Conclusion: Appendicular mass is a serious complication resulting from perforation of inflammed appendix, showing inflammatory phlegmon and/or extraluminal fluid collection with associated imaging findings. Computed tomography is highly useful in diagnosis and differentiation of appendiceal masses and deciding the management approach.

\section{INTRODUCTION}

Appendicular lump is a serious complication of untreated or inadequately treated appendicitis. The inflammed appendix is isolated by intestine and omentum from surrounding structures to contain the spread of infection leading to formation of palpable appendicular mass [1]. The inflammed appendix may perforate leading to abscess formation and even septicaemia. These patients present clinically with right iliac fossa (RIF) pain and mass which has a range of differential diagnosis including ileocaecal tuberculosis, intussusception, cecal or appendiceal neoplasm, tuboovarian mass, ectopic kidney and psoas abscess [2-5]. Multislice computed tomography (MSCT) imaging plays an important role in diagnosis and differentiation of causes of RIF pain and masses and is often the modality of choice for evaluation of these patients [3-6]. Uncomplicated cases of acute appendicitis are easy to diagnose, however, diagnosis of complicated appendicitis and appendicular 
lump is difficult and challenging. MSCT offers the advantage of direct visualization of the appendix, periappendiceal inflammatory changes and structures and also other intraabdominal organs [6] and has an important role in diagnosis and differentiation of appendicular mass.

\section{MATERIALS AND METHODS}

In this retrospective study total 56 consecutive patients (31 men and 25 women), who were diagnosed with inflammatory appendiceal masses and had undergone computed tomography (CT) examination at Smt. Kashibai Navale Medical college and GH, Pune from October 2011 to November 2015, were considered. CT scans were performed on Siemens Somatom Spirit multidetector helical CT. Oral and rectal contrast was administered in all patients to opacify the bowel. Contiguous axial CT sections were obtained before injection of iodinated intravenous contrast medium, in venous phase 45 to 60 seconds after the initial injection of contrast and in delayed phase after 5 minutes of contrast injection as per requirement. Axial source images and reformatted images were viewed and analysed in detail. Images were evaluated by two radiologists separately for inflammed appendix, appendicolith, omental and periappendiceal fat inflammation, inflammatory phlegmon, extraluminal fluid collection, bowel aggregation, bowel wall thickening, small bowel dilatation, fascial thickening and lymph nodes. Whenever there was difference in the imaging findings of the two radiologists, the opinion of the third radiologist was obtained and findings with majority consensus were considered in the final analysis. The CT findings were correlated either with the follow up clinical outcome and imaging studies in patients on conservative treatment and intraoperative and histological findings in patients who had undergone surgical intervention. The patients of right iliac fossa who were found to have other underlying pathology like tuberculosis, Crohn's disease and malignancy were excluded from the study. The institutional ethical committee cleared the study.

\section{RESULTS}

The present study included 56 patients [Table/Fig-1] diagnosed with inflammatory appendiceal masses with $31(55 \%)$ men and 25 (45\%) women (M: F ratio of 1.2:1). The mean age was 44 years (44.4 years for men and 43.6 years for women) with an age range of 4 to 79 years. The maximum number of patients (26 patients, 46\%) was in the age range of 51 to 80 years. All patients on abdominal examination had clinically palpable lump in right iliac fossa (RIF). Thirty-six patients (64\%) also gave history of RIF pain while 22 (39\%) patients had previous episodes of RIF pain. Twenty patients (35.7\%) had abdominal distension and 15 patients (27\%) each complained of fever and vomiting. The duration of symptoms prior to admission ranged from two to forty five days. Thirty five patients (62.5\%) had symptoms for more than one week duration. [Table/Fig-2] demonstrates the spectrum of CT imaging findings that were presents in these patients. The inflamed appendix could be identified in the inflammatory mass in 22 cases (39\%) with appendicolith seen in 12 cases (21\%). Suspected site of perforation could be identified in 10 out of 22 patients. Inflammatory phlegmon [Table/Fig-3A-3C] was seen in 38 cases (68 \%). Omental thickening and fat inflammation [Table/Fig-3C] was present in 46 out of 56 cases (82\%) with adjoining aggregated small bowel loops were seen in 36 cases (64\%). Extraluminal fluid collections [Table/Fig-3-5] were present in 35 patients (62.5 $\%)$ out of which enhancing walls were seen in 26 (46 \%) patients which were labelled as abscesses [Table/Fig-4,5]. The fluid collections were seen tracking in right side of pelvis in 9 patients [Table/Fig-5]. Reactive lymph node enlargement [Table/Fig-3D] was noticed in 38 cases (68\%). Adjacent ileocaecal wall thickening [Table/Fig-3B] was seen 22 cases (39 \%) with caecal thickening seen in all patients while

\begin{tabular}{|c|c|c|c|}
\hline Age in years /Sex & Male & Female & Total \\
\hline $0-10$ & 2 & 4 & 6 \\
\hline $11-20$ & 4 & 1 & 5 \\
\hline $21-30$ & 3 & 2 & 5 \\
\hline $31-40$ & 5 & 3 & 8 \\
\hline $41-50$ & 2 & 4 & 6 \\
\hline $51-60$ & 6 & 5 & 11 \\
\hline $61-70$ & 6 & 5 & 11 \\
\hline $71-80$ & 3 & 1 & 4 \\
\hline Total & 31 & 25 & 56 \\
\hline
\end{tabular}

[Table/Fig-1]: Age and Sex-wise distribution of patients.

\begin{tabular}{|c|l|c|}
\hline Sr. No. & Imaging finding & $\begin{array}{c}\text { No. of patients } \\
(\%) \quad \mathrm{n}=56\end{array}$ \\
\hline 1. & Inflammed appendix & $22(39.3)$ \\
\hline 2. & Appendicolith & $12(21.4)$ \\
\hline 3. & $\begin{array}{l}\text { Omental/ periappendiceal } \\
\text { fat inflammation }\end{array}$ & $46(82.1)$ \\
\hline 4. & Phlegmon & $38(67.8)$ \\
\hline 5. & Extraluminal Fluid collection & $35(62.5)$ \\
\hline 6. & Extraluminal air pockets & $31(55.3)$ \\
\hline 7. & Bowel aggregation & $36(64.3)$ \\
\hline 8. & Bowel wall thickening & $22(39.3)$ \\
\hline 9. & Small bowel dilatation & $20(35.7)$ \\
\hline 10. & Fascial thickening & $32(57.1)$ \\
\hline 11. & Lymph nodes & $38(67.8)$ \\
\hline [Table/Fig-2]: Spectrum of CT findings in inflammatory appendiceal \\
masses.
\end{tabular}



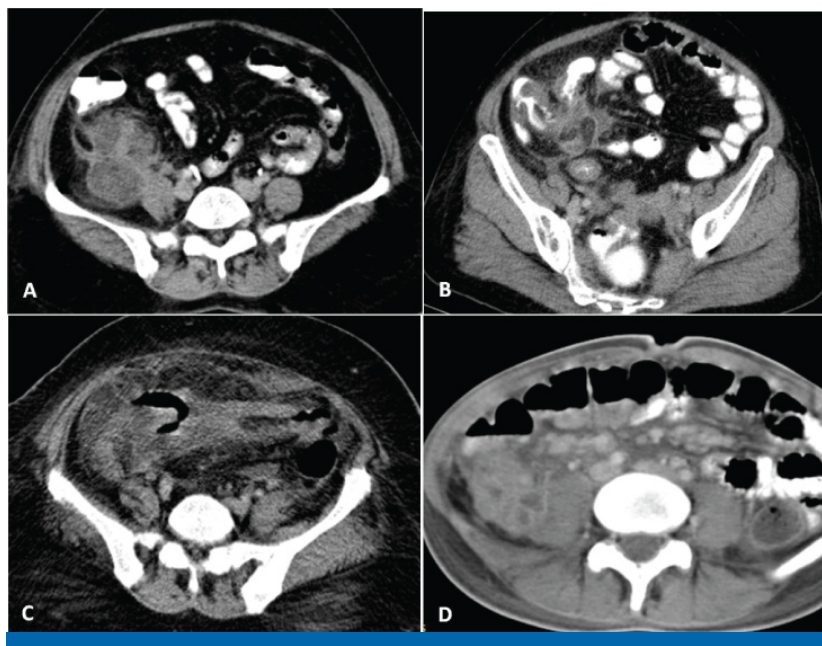

[Table/Fig-3]: Appendicular phlegmonous masses (A to C) showing abscesses (A), ill-defined fluid collections and bowel wall thickening (B), extraluminal air, omental inflammation and thickening (C). Another case of appendicular lump shows heterogeneous inflammatory mass with lymphadenopathy (D).
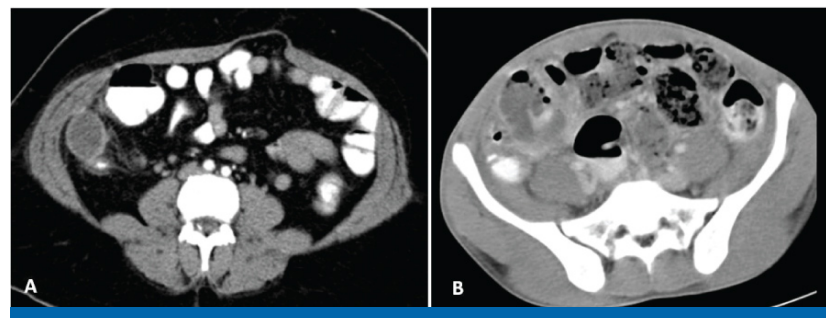

[Table/Fig-4]: Post contrast axial CT image (A) reveals appendicular abscess with appendicolith. Axial CT image of another patient (B) showing appendicular abscess with extraluminal air pockets.

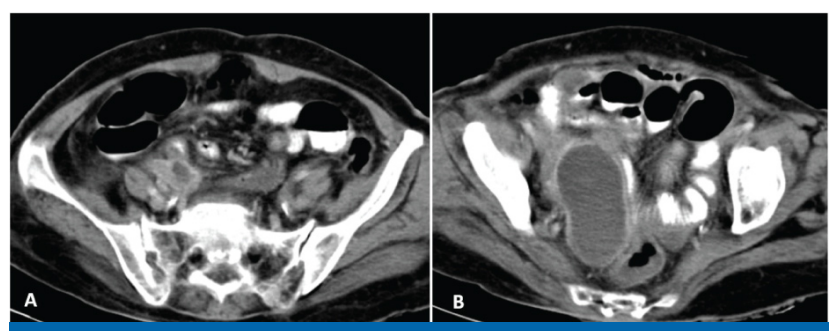

[Table/Fig-5]: Post contrast axial CT images showing perforated appendix with abscess along tip of the appendix (A), extending in right side of pelvis (B).

terminal ileal thickening seen in 15 patients. Small bowel dilatation was present in 20 (35.7\%) patients, which relieved with conservative treatment in 17 patients while 3 patients underwent laparotomy. The most common location of the appendiceal inflammatory mass was inferomedial to the caecum, seen in 39 patients (69.6\%), followed by lateral to caecum seen in 8 patients (14.2\%), while 9 patients (16\%) had more inferiorly located masses with varying extension in pelvis. Extraluminal air pockets [Table/Fig-3C,4B] were seen in 31 patients (55\%). All patients had been followed up clinically and with imaging as per indication to assess the evolution of inflammatory mass. Patients were treated with combinations of broad spectrum antibiotics, bed rest, nasogastric suction and intravenous fluids. Out of 35 patients with extraluminal fluid collections, 24 were drained percutaneously under image guidance which included 15 patients diagnosed as abscess. In five patients, the collections were small in size and resolved with conservative treatment. Nineteen patients including four patients with large abscesses and pelvic extensions underwent laparotomy. Four patients required second intervention to drain intraabdominal abscesses. Seven patients developed post operative complications which included skin wound infection in four patients, generalized peritonitis in two patients and paralytic ileus in three patients. Patients who had undergone surgical exploration, the definitive diagnosis was provided by intraoperative findings and histological examination.

\section{DISCUSSION}

An appendicular mass, a serious complication of untreated or inadequately treated appendicitis, occurs when greater omentum, mesentery and small bowel loops become adherent to the inflammed perforated appendix walling off the pus spillage and spread of peritoneal contamination $[1,7]$. This results in formation of appendicular mass which may be an inflammatory plegmonous mass or an appendiceal abscess $[7,8]$. Phlegmon is seen as an inflammatory mass consisting of the inflammed appendix, its adjacent viscera and the greater omentum on CT, while abscess appears as an extraluminal fluid collection or pus-containing periappendiceal mass [7-9]. Inflammatory phlegmonous mass was seen in 38 cases (68\%) in our study and extraluminal fluid collections were seen in 35 patients (62.5\%), out of which collections with enhancing walls seen in 26 cases (46 \%) were labelled as abscesses. The fluid collections were seen tracking in right side of pelvis in 9 patients [Table/Fig-5]. The abscess is most often located in the proximity of the appendix leading to formation of inflammatory mass adjacent to the caecum and terminal ileum. The abscesses, depending on the location and length of the appendix, have been described in the left lower quadrant, the right flank, the anterior abdominal wall, the lesser sac, and in the subhepatic and subdiaphragmatic spaces [9]. Contrast enhanced CT may demonstrate the ruptured appendix seen as defects in the wall of the appendix; however, abnormal appendix may not be demonstrated in many of the patients with appendiceal mass. The inflamed appendix could be identified in the inflammatory mass in 22 cases (39\%) in our study and suspected site of perforation could be identified in 10 out of 22 patients [Table/Fig-5A]. The appendicolith may be present outside the lumen of appendix, located in an inflammatory mass or abscess. The presence of appendicolith with periappendiceal abscess 
or phlegmon helps in making the diagnosis of ruptured appendix in some patients and was seen in 12 cases (21\%) in this study. Associated imaging findings of extraluminal air, ileocaecal wall thickening, localised lymphadenopathy, aggregated small bowel loops and small-bowel obstruction are also seen in many patients of appendicular mass [7-9]. Extra luminal air pockets [Table/Fig-3C,4B] were seen in 31 patients (55\%) in present study. Extraluminal air within fluid collection suggests either perforation or infection due to gas forming organisms [10]. Ileocecal wall thickening [Table/Fig3B] was seen 22 cases (39\%) with adjoining aggregated small bowel loops were seen in 36 cases (64\%) in our study. Omental thickening and fat inflammation [Table/Fig-3C] was present in 46 out of 56 cases (82\%) and reactive lymph node enlargement [Table/Fig-3D] was noticed in 38 cases (68\%). Small bowel dilatation was present in 20 (35.7\%) patients, which relived with conservative treatment in 17 patients while 3 patients underwent laparotomy.

Right iliac fossa mass, apart from appendicular mass, has a range of differentials including ileocecal tuberculosis, intussusception, cecal or appendiceal neoplasm, tuboovarian mass, ectopic kidney, right-sided colitis and psoas abscess and CT imaging plays an important role in their diagnosis and differentiation [3-6]. Crohn's disease commonly involves the terminal ileum causing bowel wall thickening, increased attenuation of mesenteric fat, mesenteric fibrofatty proliferation and mesenteric lymphadenopathy on CT imaging with abscess and fistula formation seen in complicated cases $[3,5]$. Visualization of normal appendix with inflammation located away for appendix, bowel wall thickening with mural stratification and presence of skip lesions helps in its differentiation from appendicular mass [5,11]. Ileocecal tuberculosis, most common site of gastrointestinal tuberculosis, is seen as circumferential wall thickening of the cecum, terminal ileum and ileocecal valve. Presence of associated necrotic mesenteric lymphadenopathy with rim enhancement on contrast enhanced CT is useful in diagnosis of lleocecal tuberculosis [12,13]. The extent of colonic wall thickening is more in cases of colitis than appendicitis and is seen as circumferential long segment wall thickening of the colon with adjacent fat stranding [3]. Intussusception, more common in children, appears as an abnormal target like mass of characteristic 'bowel in bowel' configuration in CT with or without small bowel obstruction [3]. Tubo-ovarian masses and cysts may be complicated by rupture, torsion, or haemorrhage and may present as painful RIF mass. The presence of a complex adnexal mass extending in right iliac fossa with hemorrhagic free fluid on CT helps in the diagnosis [3]. CT findings of ovarian mass with areas of fat attenuation or fat-fluid levels, calcification and teeth can give conclusive diagnosis in ovarian dermoid [14]. Cecal and appendicular neoplasms, encountered in elderly patients, show nodular and asymmetric thickening of the cecum or appendiceal base [5]. Small appendicular neoplasm can occlude the appendicular lumen, causing secondary appendicitis and may be seen as enhancing intraluminal lesion on contrast enhancement CT. Treatment options for appendiceal mass ranges from conservative to aggressive surgical approaches [8,15-18]. Immediate appendicectomy following inflammatory mass resolution is an emerging alternative with the advent of antibiotics designed to prevent post operative infection and complications [17]. It is important to diagnose presence of appendiceal abscess on CT imaging for which initial percutaneous drainage may be considered an appropriate treatment option $[15,18]$. The percutaneous abscess drainage and interval appendicectomy is recommended by some authors due to lower postoperative complication rate and shorter hospital stay [15,18]. Lui et al., [19] opined that the presence of significant fat stranding associated with fluid accumulation, inflammatory mass or localized abscess in preoperative CT scanning significantly increased the possibility of conversion from laparoscopic to open appendectomy. These factors make the preoperative CT assessment of perforated appendix with associated abscess or phlegmon formation critical in determining the therapeutic options, including nonsurgical treatment and in estimating the risk of complications $[9,19,20]$.

\section{CONCLUSION}

Appendicular mass is a serious complication resulting from perforation of inflammed appendix, showing inflammatory phlegmon and/or extraluminal fluid collection with associated imaging findings. These patients present clinically with palpable right iliac fossa which has a range of differential diagnosis and computed tomography has an important role in their diagnosis and differentiation. The preoperative CT assessment of inflammatory appendiceal masses is critical in determining the therapeutic options and in estimating the risk of complications. CT also helps to diagnose presence of appendiceal abscess which often requires immediate percutaneous or open surgical drainage.

\section{REFERENCES}

[1] Bailey and Love's Short Practice of Surgery edited by Norman S. Williams, Christopher K.K. Bulstrode, P. Ronan O'Connell. $26^{\text {th }}$ edition. Taylor \& Francis Group, LLC, CRC press 2013. Pg. 1202

[2] Sargar KM, Siegel MJ. Sonography of acute appendicitis and its mimics in children. The Indian Journal of Radiology \& Imaging. 2014; 24(2):163-70.

[3] Sharma M, Agrawal A. Pictorial essay: CT scan of appendicitis and its mimics causing right lower quadrant pain. The Indian Journal of Radiology \& Imaging. 2008;18(1):80-89.

[4] Purysko AS, Remer EM, Filho HM, Bittencourt LK, Lima RV, Racy DJ. Beyond appendicitis: common and uncommon 
gastrointestinal causes of right lower quadrant abdominal pain at multidetector CT. Radiographics. 2011;31(4):927-47.

[5] Leite PN, Pereira JM, Cunha R, Pinto P, Sirlin C. CT evaluation of appendicitis and its complications: imaging techniques and key diagnostic findings. AJR Am J Roentgenol. 2005; 185(2):40617.

[6] Whitley S, Sookur P, McLean A, Power N. The appendix on CT. Clin Radiol. 2009 ; 64(2):190-99.

[7] Gupta AK, Srivastava DP, Khandelwal N, Chowdhury V. Diagnostic Radiology: Gastrointestinal and Hepatobiliary Imaging $3^{\text {rd }}$ Ed. Jaypee Brothers Medical Publishers. (2009). Ch 11. Pg 194-96.

[8] Tannoury $\mathrm{J}$ and Abboud B. Treatment options of inflammatory appendiceal masses in adults. World J Gastroenterol. 2013; 19(25): 3942-50.

[9] Horrow MM, White DS, Horrow JC. Differentiation of perforated from non perforated appendicitis at CT. Radiology. 2003;227(1):46-51.

[10] Birnbaum BA, Wilson SR. Appendicitis at the Millenium. Radiology. 2000; 215:337-48.

[11] Yu J, Fulcher AS, Turner MA, Halvorsen RA. Helical CT evaluation of acute right lower quadrant pain: Part I, Common mimics of appendicitis. AJR Am J Roentgenol. 2005:;184:1136-42.

[12] Zhao J, Cui MY, Chan T et al. Evaluation of intestinal tuberculosis by multi-slice computed tomography enterography. BMC Infectious Diseases. 2015; 15:577.

\section{AUTHOR(S): \\ 1. Dr. Varsha Rangankar \\ 2. Dr. Abhijit Pawar \\ 3. Dr. Aditi Dongre \\ 4. Brig Dr. Hariqbal Singh}

\section{PARTICULARS OF CONTRIBUTORS:}

1. Associate Professor, Department of Radiology, Smt. Kashibai Navale Medical College \& GH, Pune, India.

2. Associate Professor, Department of Radiology, Smt. Kashibai Navale Medical College \& GH, Pune, India.

3. Lecturer, Department of Radiology, Smt. Kashibai Navale Medical College, Pune, India.
[13] Kedia S, Sharma R, Nagi B, Mouli VP, Aananthakrishnan A, Dhingra et al. Computerized tomography-based predictive model for differentiation of Crohn's disease from intestinal tuberculosis. Indian J Gastroenterol. 2015;34(2):135-43.

[14] Yu J, Fulcher AS, Turner MA, Halvorsen RA. Helical CT evaluation of acute right lower quadrant pain: part II, uncommon mimics of appendicitis. AJR Am J Roentgenol. 2005; 184(4):1143-49.

[15] Hogan MJ. Appendiceal abscess drainage. Tech Vasc Interv Radiol. 2003; 6:205-14.

[16] Tekin A, Kurtoğlu HC, Can I, Oztan S. Routine interval appendectomy is unnecessary after conservative treatment of appendiceal mass. Colorectal Dis. 2008;10:465-68.

[17] Meshikhes AW. Management of appendiceal mass: controversial issues revisited. J Gastrointest Surg. 2008;12(4):767-75.

[18] Brown CV, Abrishami M, Muller M, Velmahos GC. Appendiceal abscess: immediate operation or percutaneous drainage? Am Surg. 2003; 69:829- 32

[19] Liu SI, Siewert B, Raptopoulos V, Hodin RA. Factors associated with conversion to laparotomy in patients undergoing laparoscopic appendectomy. J Am Coll Surg. 2002; 194:298305.

[20] Oliak D, Sinow R, French S, Udani VM, Stamos MJ. Computed tomography scanning for the diagnosis of perforated appendicitis. Am Surg. 1999; 65:959-64.

4. Professor and Head, Department of Radiology, Smt. Kashibai Navale Medical College \& GH, Pune, India.

\section{NAME, ADDRESS, E-MAIL ID OF THE CORRESPONDING AUTHOR:}

Dr. Varsha Rangankar,

Associate Professor, Department of Radiology,

SKN Medical College and GH, Pune-411 041, India.

E-mail: varsharangankar@gmail.com

FINANCIAL OR OTHER COMPETING INTERESTS:

None.

Date of Publishing: Apr 01, 2016 